\title{
Prevención y control de Covid-19 en Instituciones de Larga Estancia para ancianos
}

Prevenção e Controle da Covid-19 em Instituições de Longa Permanência para idosos

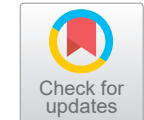

Prevention and control of Covid-19 in Long-Time Institutions for elderly people

Como citar este artículo: Felipe, Sarah Giulia Bandeira; Silva, Cynthia Roberta Dias Torres; Carvalho, Khelyane Mesquita de; Gouveia, Marcia Teles de Oliveira; Figueiredo, Maria do Livramento Fortes. Prevención y control de Covid-19 en Instituiciones de Larga Estancia para ancianos. Revista Cuidarte. 2021;12(1):e1380.

http://dx.doi.org/10.15649/cuidarte.1380

Revista Cuidarte

doit. Rev Cuid. Ene. - Abril. 2021; 12(1): e1380

http://dx.doi.org/10.15649/cuidarte.1380

E-ISSN: 2346-3414

(1) Sarah Giulia Bandeira Felipe

(1) Cynthia Roberta Dias Torres Silva²

(1) Khelyane Mesquita de Carvalho 3

(1) Marcia Teles de Oliveira Gouveia ${ }^{4}$

(1) Maria do Livramento Fortes Figueiredo

1 Universidade Federal do Piaui- UFPI. Campus Universitário Ministro Petrônio Portella, Teresina, Piauí, Brasil. Autor Correspondiente E-mail: sarinhagbf@hotmail.com

2 Universidade Federal do Piaui- UFPI. Campus Universitário Ministro Petrônio Portella, Teresina, Piauí, Brasil. E-mail: cynthiarobertatorres@gmail.com

3 Universidade Federal do Piaui- UFPI. Campus Universitário Ministro Petrônio Portella, Teresina, Piauí, Brasil.

E-mail:khleyanemc@gmail.com

4 Universidade Federal do Piaui- UFPI. Campus Universitário Ministro Petrônio Portella, Teresina, Piauí Brasil. E-mail: marcia06@gmail.com

5 Universidade Federal do Piaui- UFPI. Campus Universitário Ministro Petrônio Portella, Teresina, Piauí, Brasil. E-mail: liff@ufpi.edu.br
Estimada Editora,

COVID-19 es una enfermedad causada por el virus del Síndrome Respiratorio Agudo Severo Coronavirus 2 (SARS-CoV-2), es una emergencia de salud pública internacional. En Brasil, el número de muertes llegó a 13.944, de las cuales el 69\% eran personas mayores. La vulnerabilidad de los ancianos a COVID-19 puede explicarse por la inmunosenescencia del envejecimiento y la presencia de comorbilidades ${ }^{1}$, que tiende a maximizarse en entornos de vivienda colectiva como las Instituciones de Atención a Larga Estancia (IALE) debido a la rápida capacidad de propagar enfermedades infecciosas.

Los datos preliminares indican que la mitad de las muertes relacionadas con el coronavirus en Europa fueron de residentes de centros de salud. Al confrontar estos datos con la realidad de las IALE en Brasil, existe una preocupación urgente por la reestructuración de la atención y los servicios, Los datos preliminares indican que la mitad de las muertes relacionadas con el coronavirus en Europa fueron de residentes de centros de salud. Al confrontar estos datos con la realidad de las IALE en Brasil, existe una preocupación urgente por la reestructuración de la atención y los servicios, ya que son lugares predominantemente filantrópicos para acoger a personas mayores vulnerables, frágiles y dependientes.

ya que son lugares

predominantemente filantrópicos para acoger a personas mayores vulnerables, frágiles y dependientes.
Recibido: 7 de junio de 2020 Aceptado: 10 de diciembre de 2020 Publicado:19 de febrero de 2021 $\square *$ Correspondencia

Sarah Giulia Bandeira Felipe

E-mail:sarinhagbf@hotmail.com 
En ausencia de información resumida sobre este tema, el Grupo de Estudio sobre la Multidimensionalidad del Envejecimiento, la Salud y la Enfermería (GEMESE) de la Universidad Federal de Piauí, tuvo como objetivo enumerar las directrices y recomendaciones para la prevención y el control de COVID-19 en IALE, puesto a disposición por Agencias Federales Brasileñas, como subsidio a las políticas públicas intersectoriales para la protección de las personas mayores institucionalizadas ${ }^{2-4}$.

Como medida inicial, se señala la necesidad de un diagnóstico situacional, especificando la naturaleza, la capacidad, el perfil de la población y el personal de cada institución; seguido de acciones formativas y educativas. Con respecto a las relaciones sociales, se recomienda suspender las visitas externas, que pueden ser proporcionadas por telecomunicaciones.

Para proporcionar el mantenimiento y la optimización de la atención integral, está indicada la evaluación multidimensional de los ancianos, evitando agravaciones o agravamiento de afecciones crónicas que pueden causar deterioro o comprometer la autonomía e independencia. Los ejercicios físicos, las actividades de socialización colectiva, la promoción de hábitos saludables y el control de la medicación deben mantenerse adoptando las medidas de distancia recomendadas.

Entre las medidas de prevención de contagio, se destacan las siguientes: limpieza de materiales recibidos en la entrada, limpieza de manijas de puertas, manijas de sillas de ruedas, pasamanos y pasillos con solución de alcohol líquido al 70\% dos veces al día; uso de equipo de protección personal y orientación sobre cómo proceder antes, durante y después de salir de ILPI, lavado de manos adecuado, implementación de medidas de barrera con la instalación de alfombras o paños empapados con solución de hipoclorito de sodio al $30 \%$ en todas las entradas, asociado a medidas generales de prevención.

Para la detección temprana, la presencia de signos y síntomas sugestivos debe controlarse diariamente. Es aconsejable que toda persona anciana sintomática use una máscara y permanezca aislada en una habitación privada bien ventilada, preferiblemente con baño, durante al menos 14 días. Además, se enfatiza la necesidad de designar a un solo empleado para que brinde toda la atención diaria.

La hospitalización se justifica por criterios clínicos de gravedad, como la presencia de síntomas gripales o tos, dificultad para respirar o dolor de garganta agregado a la saturación de $02<95 \%$; signo de dificultad respiratoria o aumento de la frecuencia respiratoria basal; empeoramiento de condiciones crónicas; hipotensión delirio; interrupción de la ingesta de alimentos; y deterioro de la marcha. Para regresar, la persona de edad avanzada debe presentar resultados positivos en la prueba de cura inmunológica (lgG) después de 14 días de hospitalización con 72 horas sin signos clínicos de descompensación.

En este contexto, la importancia de las acciones preventivas estratégicas y la promoción de una cultura de seguridad se refuerza a través de información y directivas coherentes, con base científica, que favorecen la mejora de las políticas públicas de atención a largo plazo, la toma de decisiones y la asignación. recursos de las esferas responsables de la respuesta de emergencia a la pandemia de COVID-19.

En este contexto, la importancia de las acciones preventivas estratégicas y la promoción de una cultura de seguridad se refuerza a través de información y directivas coherentes, con base científica, que favorecen la mejora de las políticas públicas de atención a largo plazo, la toma de decisiones y la asignación. recursos de las esferas responsables de la respuesta de emergencia a la pandemia de COVID-19. 
Financiación: No hay financiación

Conflictos de Intereses: No hay conflictos de intereses

Agradecimientos: À Universidade Federal do Piauí-UFPI

\section{Referencias}

1. CDC - Centers for Disease Control and Prevention. Severe Outcomes Among Patients with Coronavirus Disease 2019 (COVID-19) — United States, February 12-March 16, 2020. MMWR Morb Mortal Wkly Rep 2020;69:343-346. http://dx.doi.org/10.15585/mmwr.mm6912e2

2. Brasil. Agência Nacional de Vigilância Sanitária - ANVISA. Nota técnica no 05/2020 GVIMS/GGTES. Orientações para a Prevenção e Controle de Infecções pelo novo coronavírus (SARS-Cov-2) em Instituições de Longa Permanência para Idosos (ILPI). Brasília, 24 de março de 2020. Available from: https://www20.anvisa.gov.br/segurancadopaciente/index.php/ alertas/item/nota-tecnica-n-05-2020-gvims-ggtes-anvisa-orientacoes-para-a-prevencao-eo-controle-deinfeccoes-pelo-novo-coronavirus-sars-cov-2-ilpi Acesso em: 27 abr. 2020.

3. Brasil. Ministério da Saúde. Secretária de Atenção Primária a Saúde. Nota técnica No 8/2020-COSAPI/CGCIVI/DAPES/SAPS/MS. Preveção e controle de infecções pelo novo coronavírus (SARS-Cov-2) a serem adotadas na Instituições de Longa Permanência de Idosos (ILPI). Brasília, 2020. Available from http://189.28.128.100/dab/docs/portaldab/documentos/ notatecnica82020COSAPICGCIVIDAPESSAPSMS02abr2020COVID-19.pdf Acesso em 29 abr. 2020.

4. Brasil. Ministério da Saúde. Secretária de Atenção Primária a Saúde. Nota técnica No 8/2020-COSAPI/CGCIVI/DAPES/SAPS/MS. Preveção e controle de infecções pelo novo coronavírus (SARS-Cov-2) a serem adotadas na Instituições de Longa Permanência de Idosos (ILPI). Brasília, 2020. Available from http://189.28.128.100/dab/docs/portaldab/documentos/ notatecnica82020COSAPICGCIVIDAPESSAPSMS02abr2020COVID-19.pdf Acesso em 29 abr. 2020. 